\title{
Biosynthesis of Gold Nanoparticles by Biotechnologically Important Active and Inactive Form of the Ascomycetes - Pichia membranifaciens and Debaryomyces hansenii
}

\author{
Alok Pani and Soon-Il Yun* \\ Department of Food Science and Technology, College of Agriculture and Life Sciences, Chonbuk National University, 567 Baekje-daero, deokjin-gu, Jeonju-si, \\ Jeollabuk-do 54896, Republic of Korea
}

Correspondence to:

Soon-Il Yun

Department of Food Science and Technology

College of Agriculture and Life Sciences

Chonbuk National University

567 Baekje-daero, deokjin-gu, Jeonju-si

Jeollabuk-do 54896, Republic of Korea

Tel: +82-63-270-2566

E-mail: siyun@jbnu.ac.kr

Received: February 24, 2016

Accepted: May 11, 2016

Published: May 13, 2016

Citation: Pani A, Yun SI. 2016. Biosynthesis of Gold Nanoparticles by Biotechnologically Important Active and Inactive Form of the Ascomycetes - Pichia membranifaciens and Debaryomyces hansenii. J Food Chem Nanotechnol 2(2): 92-96.

Copyright: ( 2016 Pani and Yun. This is an Open Access article distributed under the terms of the Creative Commons Attribution 4.0 International License (CC-BY) (http://creativecommons. org/licenses/by/4.0/) which permits commercial use, including reproduction, adaptation, and distribution of the article provided the original author and source are credited.

Published by United Scientific Group

\begin{abstract}
The two biotechnologically important Ascomycetes, Pichia membranifaciens and Debaryomyces hansenii, were tested (in active and inactive form), in the present study for efficient bioreduction of trivalent aurum to gold nanoparticles. Strong surface plasmon resonance was observed, between 530 and $540 \mathrm{~nm}$, from the gold nanoparticles recovered from the test Ascomycetes. The characterization of the gold nanoparticles thus formed was performed by HR-TEM, FE-SEM, FTIR spectroscopy. Comparing the active and inactive form of the two Ascomycetes, the inactive form showed better productivity and recovery of gold nanoparticles. It was also found that $P$. membranifaciens is more productive in terms of time, quality and quantity, than $D$. bansenii. The nanoparticles were relatively monomorphic in P. membranifaciens than in D. bansenii. The present study also presents a promising outlook towards the effective recycling of the bioactive wastes.
\end{abstract}

\section{Keywords}

Gold nanoparticles, UV-visible analysis

\section{Introduction}

Synthesis of nanoparticles of noble metals is of enormous importance due to their biomedical, pharmaceutical, optical, electronic and chemical properties [1-9]. Microbial interactions with metals have been explored for biotechnological application such as ore leaching and metal recovery [10-12]. However, only in the recent past, microbes have gained significant attention for the synthesis of metal nanoparticles. Different microorganisms such as bacteria and fungi from different habitats and nutritional modes have been employed for the synthesis of metal nanoparticles either intra- or extra-cellular [13]. Most of the research in this field has been concentrated on the synthesis of noble metal nanoparticles such as gold and silver $[14,15]$. Microorganisms have been a preferred source of synthesis due to ease of culturing, manipulation and downstream processing [13]. Microbes including simple prokaryotes to complex eukaryotic have been explored for the fabrication of nanoparticles. Among them, the yeasts and fungi are more interesting for the biosynthesis of nanoparticles due to the large amounts of secreted proteins and high yields of biomass, which make them more promising for industrial AuNPs production [13-16].

The conventional processes for nanoparticle synthesis involve top-down or bottom-up approaches usually employing physical or chemical protocols. The "Top-down" approach begins with a specific structure which is decreased in size gradually. On the other hand, the "Bottom-up" approach involves formation of nanostructures through the self-assembly of atoms and molecules. However, such 
processes are often energy-demanding and cost-intensive [17] Moreover, the chemicals used in such procedures are toxic and the size of the nanoparticles is difficult to control. In view of these problems, biological systems have emerged as effective alternatives for the clean and green synthesis of nanostructures [15].

In a previous study conducted by our group, we have already explored the intracellular and extracellular gold nanoparticles synthesis ability of Aspergillus oryzae var. viridis [18]. It is an economically important fungus used for production of rice koji in Japan. Continuing the same work, here we explore the two ascomycetes, Pichia membranifaciens and Debaryomyces hansenii, known as antagonists against diverse group of phyto-pathogens infecting the grape plant and are thus used as bio-control agents [19, 20]. Debaryomyces hansenii has biotechnological potential and utility in diversity of areas [21, 22]. This paper describes the conditions for the synthesis of AuNPs (by Live and inactive cells) and their characterization by a variety of analytical techniques.

\section{Materials and Methods}

\section{Materials}

$\mathrm{HAuCl}_{4} \cdot 3 \mathrm{H}_{2} \mathrm{O}$ (99\%) was supplied by Sigma-Aldrich. GPY agar/ broth (Glucose Peptone Yeast extract), YPD broth (Yeast extract Peptone Dextrose Broth) were supplied by Becton, Dickinson and Company (BD), USA. Pichia membranifaciens (KCTC: 17999), Debaryomyces bansenii (KCTC: 17995) were supplied by Korean Collection for Type Cultures, Daejeon, Republic of Korea.

\section{Yeast growth condition and biomass production}

Stock culture of $P$. membranifaciens and $D$. hansenii was prepared on GPY agar plate. For production of biomass, the organisms were grown aerobically in sterile YPD broth and GPY broth, respectively. The flasks were inoculated and incubated on orbital shaker at $25^{\circ} \mathrm{C}$ and $230 \mathrm{rpm}$. The culture was transferred equally to $50 \mathrm{~mL}$ falcon tubes (For each strain), after 24 hours of incubation. The yeast biomass was harvested by centrifugation $\left(6000 \mathrm{~g}, 10 \mathrm{~min}\right.$ at $\left.10{ }^{\circ} \mathrm{C}\right)$ and the pellet was washed thrice to remove residual glucose. The washed pellets were re-suspended in $10 \mathrm{~mL}$ distilled water and 3 of the 6 tubes containing the live cell biomass were autoclaved (121 ${ }^{\circ} \mathrm{C}, 15$ psi, $\left.15 \mathrm{~min}\right)$.

\section{Synthesis and recovery of gold nanoparticles}

AuNPs were synthesized by incubating the live and autoclaved yeast biomass in $1 \mathrm{mM} \mathrm{HAuCl}$ solution on orbital shaker at $25{ }^{\circ} \mathrm{C}$ and $250 \mathrm{rpm}$. The total volume of reaction mixture was $50 \mathrm{~mL}$. After synthesis the reaction tubes were centrifuged $\left(6000 \mathrm{~g}, 10 \mathrm{~min}\right.$ at $\left.10{ }^{\circ} \mathrm{C}\right)$, and the supernatant containing only AuNPs was collected and used for characterization. Visual changes and UV-visible spectra were recorded. All experiments were carried out in triplicates with positive (Only biomass suspended in water) and negative controls (Only $1 \mathrm{mM} \mathrm{HAuCl}_{4}$ ).

\section{Characterization of the gold nanoparticles}

The reaction mixtures were monitored over a period of time and visual observations were recorded. A Shimadzu UV1800 spectrophotometer was used for UV-visible spectroscopy measurements. Washed yeast cells were coated onto glass pieces and thoroughly dried. XRD measurements of thin films of yeast cells exposed to $\mathrm{HAuCl}_{4}$ were carried out under vacuum combined with energy dispersive X-ray spectroscopy (EDX) (JEOL JSM-5410LV) and X-ray diffractograms using D/ Max 2005, Rigaku. FE-SEM analysis was performed on airdried, platinum-coated cell samples using an analytical SEM (JEOL JSM-6360A). In order to determine the functional groups involved in nanoparticle synthesis, FTIR spectroscopy was carried out as described earlier [23]. For transmission electron microscope (TEM) observations, samples were coated on carbon-coated copper grids $(200 \mu \mathrm{m} \times 200 \mu \mathrm{m}$ mesh size $)$ and images were obtained on an Orius SC10002 JEM-2010 electron microscope.

\section{Results and Discussion}

\section{UV-visible analysis}

The preliminary observation and analysis of AuNP formation was based on the change in color of the reaction mixtures of $P$. membranifaciens and $D$. bansenii after 12 and 24 hours, respectively [24]. A reddish-purple transformation from straw yellow could be detected in both active and inactive cell biomass of $P$. membranifaciens and $D$. hansenii, whereas in the control tubes showed no color change [Figure 1]. A more precise detection of AuNP synthesis was done by UV-visible spectroscopy, which is an important technique to investigate surface plasmon resonance (SPR). The UV-visible spectra of AuNPs synthesized by the active and inactive cell biomass of P. membranifaciens and D. hansenii are presented in Figure 2. As shown in Figure 2, the spectral peak of the AuNPs obtained from active and inactive cell biomass of $D$. hansenii was distinctively broader than that of $P$. membranifaciens. This shows that the nanoparticles from $D$. hansenii are more polymorphic compared to that of P. membranifaciens [25].

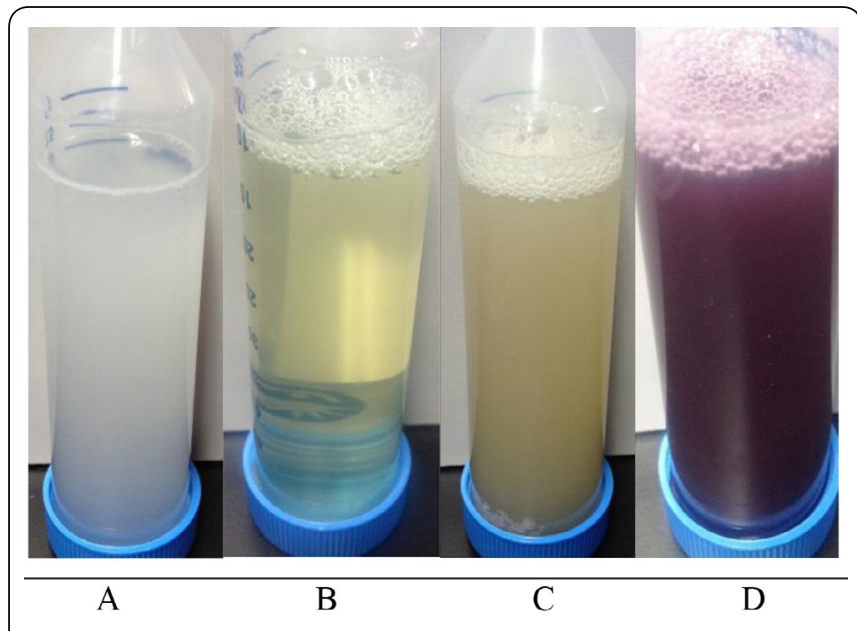

Figure 1: The preliminary visual observation and analysis. (A) Autoclaved (Inactive) cell biomass suspended in $50 \mathrm{~mL}$ distilled water. (B) $1 \mathrm{mM} \mathrm{HAuCl}_{4}$ solution $50 \mathrm{~mL}$. (C) Autoclaved (Inactive) cell biomass suspended in $50 \mathrm{~mL}$ $1 \mathrm{mM} \mathrm{HAuCl}_{4}$, imparting a straw color appearance. (D) Reaction mixture showing reddish-purple color after reduction of $\mathrm{HAuCl}_{4}$ to gold nanoparticles by the inactive cell biomass. 
The absorbance level of the nanoparticles synthesized by P. membranifaciens is higher than that of the nanoparticles synthesized by $D$. hansenii [Figure 2]. This may be due to the amount of nanoparticles that could be recovered from both the strains.

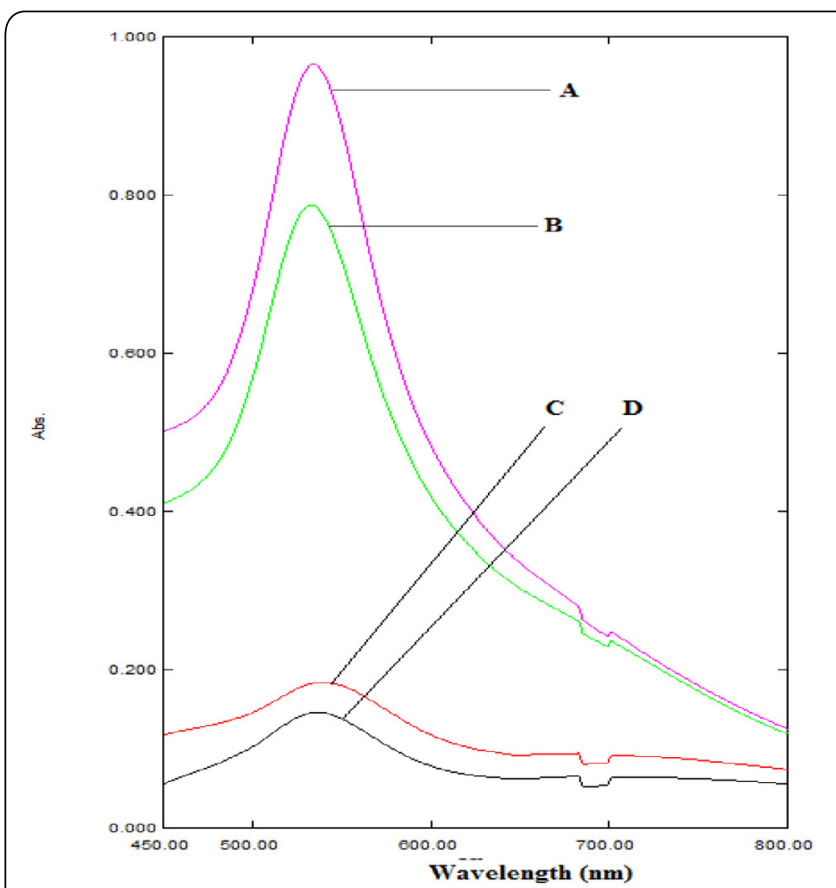

Figure 2: UV-visible spectra of gold nanoparticles recovered from reaction mixture containing, (A) inactive P. membranifaciens, (B) active P. membranifaciens, (C) inactive D. hansenii, (D) active D. hansenii.

\section{TEM, SEM and XRD analysis}

Transmission electron microscopic studies were used to analyze the particle size and morphology. Gold nanoparticles synthesized by $D$. hansenii show more polymorphism, with respect to shape and size, as compared to the ones synthesized by P. membranifaciens [Figure 3]. Figure 3B shows AuNPs of circular, triangular, pentagonal, hexagonal and irregular shaped nanoparticles [26]. Whereas, Figure 3A show TEM images of discrete gold nanoparticles of less than $50 \mathrm{~nm}$ size and they were mostly spherical-pentagonal indicating that it was possible to bio-synthesize AuNPs with satisfactory level of mono-disparity using $P$. membranifaciens. These results are in accordance to our findings in the UV-visible analysis. Nonbiological methods have been used in the past for synthesizing highly monodispersed spherical gold nanoparticles by mixing aqueous $\mathrm{HAuCl}_{4}$ and oleylamine followed by sonication at room temperature [27].

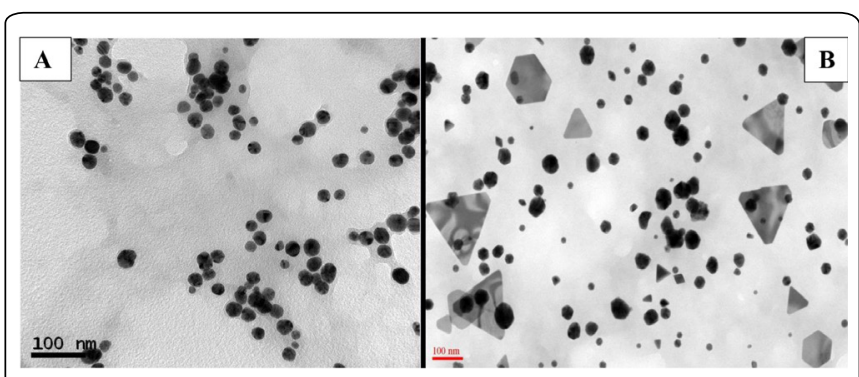

Figure 3: TEM images of gold nanoparticles synthesized by active and inactive (A) P. membranifaciens and (B) D. hansenii.
High-resolution transmission electron microscope (HRTEM) is an indispensable tool in nanotechnology. HR-TEM images of the AuNPs were used to confirm the particle morphology and size. The representative HR-TEM images of AuNPs reduced by yeasts, $P$. membranifaciens and D. hansenii, were deposited on carbon-coated copper 200 mesh grids and the morphology of the nanoparticles were found to be pentagonal, spherical, triangular and rhombohedral, with a very narrow size distribution [Figure 4]. The particles were found to be stable for more than 4 months after freeze-drying and keeping at room temperature in dry and dark condition.

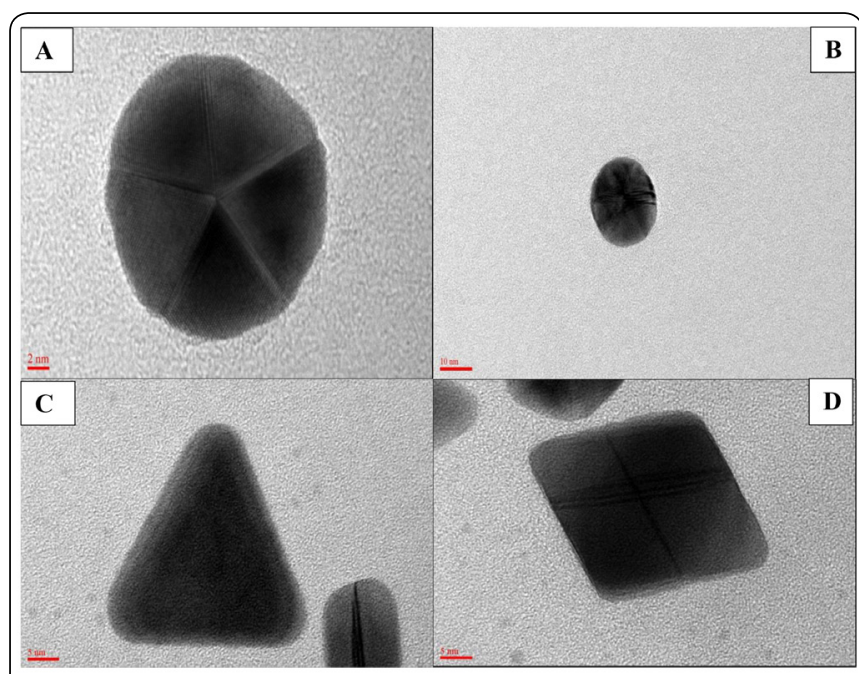

Figure 4: HR-TEM images of gold nanoparticles synthesized by active and inactive P. membranifaciens and D. hansenii showing different morphology. (A) Pentagonal (B) Spherical (C) Triangular (D) Rhombohedral.

Field emission scanning electron microscope (FE-SEM) is important technique to investigate the surface phenomenon in detail. FE-SEM has been used in this study to confirm the extracellular synthesis and also autoclaved cell mediated synthesis of AuNPs. FE-SEM analysis of the yeast cells with nanoparticles shows the maximum particle concentration outside or on the cell surface [Figure 5A and 5B]. In case of autoclaved cells, the particles could be seen on the cell

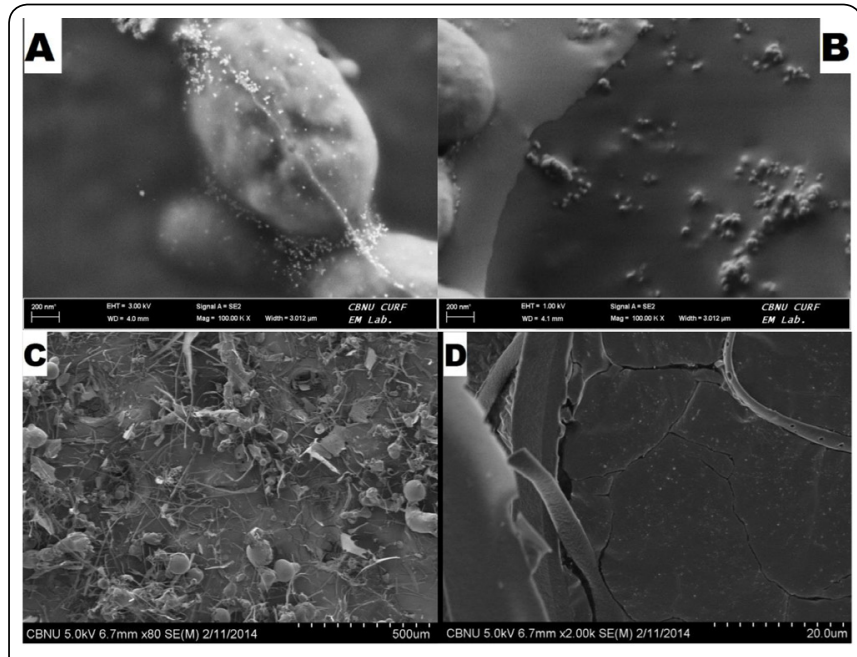

Figure 5: FE-SEM images of gold nanoparticles synthesized by yeast cells. (A) Image of yeast cells with nanoparticles on their surface. (B) Image of nanoparticles near the yeast cells. (C) Image of autoclaved yeast with nanoparticles. (D) Image of nanoparticles on the autoclaved cell debris. 
debris [Figure 5C and 5D]. This extracellular and intercellular presence of nanoparticles illustrates the extracellular synthesis of gold nanoparticles. Use of inactive fungal strain of Aspergillus oryzae is the only report of inactive cells involved in synthesis of AuNPs [18].

The representative XRD pattern of the prepared gold nanoparticles is shown in Figure 6. The synthesized AuNPs show Bragg reflections indexed to (111), (200), (220) and (311) facets of the face-centered cubic metal gold structure.

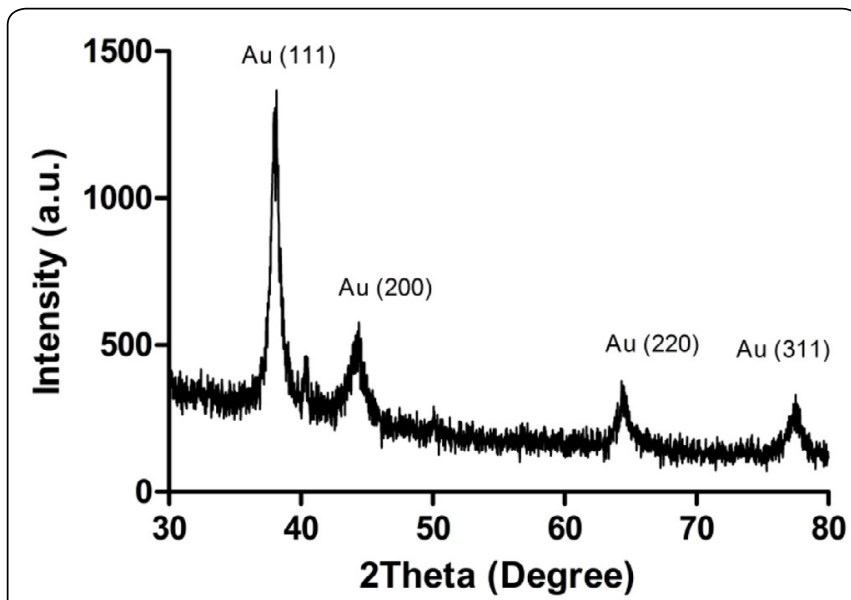

Figure 6: X-ray diffraction spectrum of gold nanoparticles synthesized by inactive cell biomass of $P$. membranifacienss.

\section{FTIR analysis}

The FTIR profiles of gold loaded, active cell biomass (blue line) and inactive cell biomass (black line), of $P$. membranifaciens is shown in Figure 7. Except for the change in the intensity of the bands, there was no major difference in the peak pattern between the biomaterials used. The peaks represent the functional groups present in the biomaterial and thus the steepness represents the difference in quantity [28]. The two peaks at 1660 and $1530 \mathrm{~cm}^{-1}$ are assigned to the amide I and II bands of proteins, respectively. Being a fact that proteins can bind to gold nanoparticles and therefore stabilization of the gold nanoparticles by surface bound proteins is a possibility [29-31]. There is also a stretching vibration of the band representing $\mathrm{NH}$ group at $3300 \mathrm{~cm}^{-1}, \mathrm{C}-\mathrm{H}$ group at $2940 \mathrm{~cm}^{-1}$ and $\mathrm{C}-\mathrm{N}$ group at $1054 \mathrm{~cm}^{-1}$ [32]. The above data suggest the involvement of $\mathrm{C}-\mathrm{N}$ and amino groups in the nanoparticle synthesis.

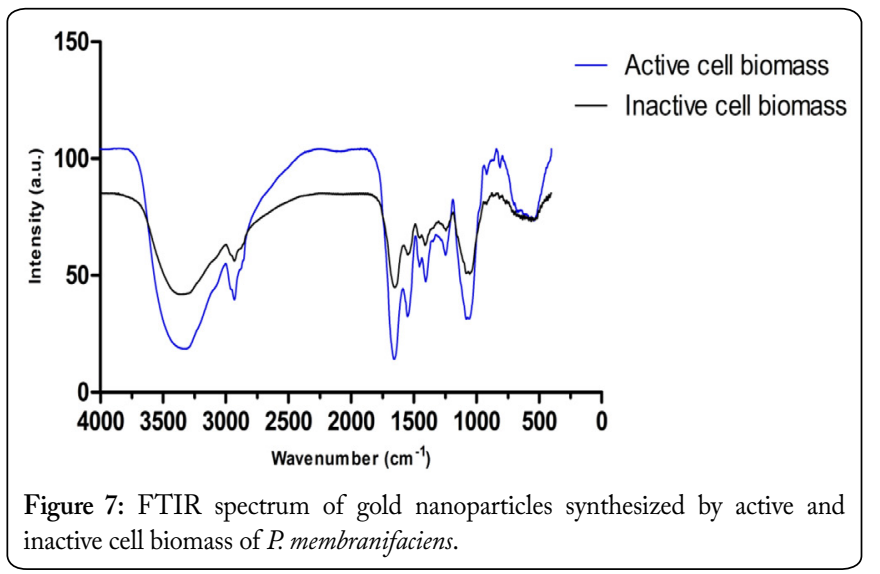

\section{Conclusion}

The present study clearly illustrates the significant role of two novel biotechnologically important Ascomycetes, in the synthesis of gold nanoparticles, in inactive and active state. Although, nanoparticles are being efficiently synthesized using chemical and physical methods, however the adverse effects of these methods sought for the discovery of novel sustainable methods. These results may open up a novel path for research communities to identify the particular component of these yeast cells responsible for nanoparticle synthesis even in inactive state. This may also lead to the industrial use of inactive or autoclaved yeast cells for production of bioactive and non-toxic gold nanoparticles.

\section{Acknowledgements}

This research was supported by Basic Science Research Program through the National Research Foundation of Korea (NRF) funded by the Ministry of Education (2013R1A1A2007953). This research was supported by funds from Chonbuk National University in 2011, Republic of Korea.

\section{References}

1. Yasui H, Takeuchi R, Nagane M, Meike S, Nakamura Y, et al. 2014. Radiosensitization of tumor cells through endoplasmic reticulum stress induced by PEGylated nanogel containing gold nanoparticles. Cancer Lett 347(1): 151-158. doi: 10.1016/j.canlet.2014.02.005

2. Cho HJ, Oh J, Choo MK, Ha JI, Park Y, et al. 2014. Chondroitin sulfate-capped gold nanoparticles for the oral delivery of insulin. International Journal of Biological Macromolecules 63: 15-20. doi: 10.1016/j.ijbiomac.2013.10.026

3. Plowman B, Ippolito SJ, Bansal V, Sabri YM, O’Mullane AP, et al. 2009. Gold nanospikes formed through a simple electrochemical route with high electrocatalytic and surface enhanced Raman scattering activity. Chem Commun 33: 5039-5041. doi: 10.1039/B910830K

4. Bansal V, O'Mullane AP, Bhargava SK. 2009. Galvanic replacement mediated synthesis of hollow Pt nanocatalysts: significance of residual $\mathrm{Ag}$ for the $\mathrm{H}_{2}$ evolution reaction. Electrochemistry Communications 11(8): 1639-1642. doi: 10.1016/j.elecom.2009.06.018

5. Bansal V, Li V, O’Mullane AP, Bhargava SK. 2010. Shape dependent electrocatalytic behaviour of silver nanoparticles. CrystEngComm 12: 4280-4286. doi: 10.1039/C0CE00215A

6. Jackson P, Periasamy S, Bansal V, Geso M. 2011. Evaluation of the effects of gold nanoparticle shape and size on contrast enhancement in radiological imaging. Australas Phys Eng Sci Med 34(2): 243-249. doi: 10.1007/s13246-011-0071-7

7. Pearson A, O'Mullane AP, Bansal V, Bhargava SK. 2010. Galvanic replacement mediated transformation of $\mathrm{Ag}$ nanospheres into dendritic $\mathrm{Au}-\mathrm{Ag}$ nanostructures in the ionic liquid $[\mathrm{BMIM}]\left[\mathrm{BF}_{4}\right]$. Chem Commun (Camb) 46(5): 731-733. doi: 10.1039/b918866e

8. Pearson A, O'Mullane AP, Bansal V, Bhargava SK. 2011. Galvanic replacement of semiconductor phase I CuTCNQ microrods with $\mathrm{KAuBr}_{4}$ to fabricate $\mathrm{CuTCNQ} / \mathrm{Au}$ nanocomposites with photocatalytic properties. Inorg chem 50(5): 1705-1712. doi: 10.1021/ic1021752

9. Abu ZS, Bansal V, McCulloch DG, Spizzirri PG, Latham K, et al. 2011. Facile, size-controlled deposition of highly dispersed gold nanoparticles on nitrogen carbon nanotubes for hydrogen sensing. Sensors and Actuators B: Chemical 160(1): 1034-1042. doi: 10.1016/j. snb.2011.09.022

10. Bansal V, Sanyal A, Rautaray D, Ahmad A, Sastry M. 2005. Bioleaching 
of sand by the fungus Fusarium oxysporum as a means of producing extracellular silica nanoparticles. Adv Mater 17(7): 889-892. doi: 10.1002/adma.200401176

11. Bansal V, Syed A, Bhargava SK, Ahmad A, Sastry M. 2007. Zirconia enrichment in zircon sand by selective fungus-mediated bioleaching of silica. Langmuir 23(9): 4993-4998. doi: 10.1021/la062535x

12. Mulligan CN, Kamali M. 2003. Bioleaching of copper and other metals from low-grade oxidized mining ores by Aspergillus niger. J Chem Technol Biotechnol 78(5): 497-503. doi: 10.1002/jctb.830

13. Bansal V, Bharde A, Ramanathan R, Bhargava SK. 2012. Inorganic materials using 'unusual' microorganisms. Adv Colloid Interface Sci 179182: 150-168. doi: $10.1016 /$ j.cis.2012.06.013

14. Narayanan KB, Sakthivel N. 2011. Green synthesis of biogenic metal nanoparticles by terrestrial and aquatic phototrophic and heterotrophic eukaryotes and biocompatible agents. Adv Colloid Interface Sci 169(2): 59-79. doi: 10.1016/j.cis.2011.08.004

15. Thakkar KN, Mhatre SS, Parikh YR. 2010. Biological synthesis of metallic nanoparticles. Nanomedicine 6(2): 257-262. doi: 10.1016/j. nano.2009.07.002

16. Kitching M, Ramani M, Marsili E. 2015. Fungal biosynthesis of gold nanoparticles: mechanism and scale up. Microb Biotechnol 8(6): 904917. doi: $10.1111 / 1751-7915.12151$

17. Zinjarde SS. 2012. Bio-inspired nanomaterials and their applications as antimicrobial agents. CYS 3(1): 74-81. doi: 10.4103/2229-5186.94314

18. Binupriya AR, Sathishkumar M, Vijayaraghavan K, Yun SI. 2010. Bioreduction of trivalent aurum to nano-crystalline gold particles by active and inactive cells and cell-free extract of Aspergillus oryzae var. viridis. J Hazard Mater 177(1): 539-545. doi: 10.1016/j. jhazmat.2009.12.066

19. Masih EI, Alie I, Paul B. 2000. Can the grey mould disease of the grape-vine be controlled by yeast?. FEMS Microbiol Lett 189(2): 233237. doi: 10.1111/j.1574-6968.2000.tb09236.x

20. Masih EI, Slezack-Deschaumes S, Marmaras I, Barka EA, Vernet G et al. 2001. Characterisation of the yeast Pichia membranifaciens and its possible use in the biological control of Botrytis cinerea, causing the grey mould disease of grapevine. FEMS Microbiol Lett 202(2): 227-232. doi: 10.1111/j.1574-6968.2001.tb10808.x

21. Breuer U, Harms H. 2006. Debaryomyces hansenii-an extremophilic yeast with biotechnological potential. Yeast 23(6): 415-437. doi: $10.1002 /$ yea. 1374
22. Fleet GH. 2007. Yeasts in foods and beverages: impact on product quality and safety. Curr Opin Biotechnol 18(2): 170-175. doi: 10.1016/j. copbio.2007.01.010

23. Bankar A, Joshi B, Kumar AR, Zinjarde S. 2010. Banana peel extract mediated synthesis of gold nanoparticles. Colloids Surf B Biointerfaces 80(1): 45-50. doi: 10.1016/j.colsurfb.2010.05.029

24. Musarrat J, Dwivedi S, Singh BR, Al-Khedhairy AA, Azam A, et al. 2010. Production of antimicrobial silver nanoparticles in water extracts of the fungus Amylomyces rouxii strain KSU-09. Bioresour Technol 101(22): 8772-8776. doi: 10.1016/j.biortech.2010.06.065

25. Chen H, Kou X, Yang Z,Ni W,WangJ.2008. Shape-and size-dependent refractive index sensitivity of gold nanoparticles. Langmuir 24(10): 5233-5237. doi: 10.1021/la800305j

26. Das SK, Das AR, Guha AK. 2010. Microbial synthesis of multishaped gold nanostructures. Small 6(9): 1012-1021. doi: 10.1002/ smll.200902011

27. Polavarapu L, Xu QH. 2009. A simple method for large scale synthesis of highly monodisperse gold nanoparticles at room temperature and their electron relaxation properties. Nanotechnology 20(18): 185606. doi: 10.1088/0957-4484/20/18/185606

28. Binupriya AR, Sathishkumar M, Ku CS, Yun SI. 2010. Sequestration of Reactive Blue 4 by free and immobilized Bacillus subtilis cells and its extracellular polysaccharides. Colloids Surf B Biointerfaces 76(1): 179185. doi: 10.1016/j.colsurfb.2009.10.031

29. Sastry M, Ahmad A, Khan MI, Kumar R. 2003. Biosynthesis of metal nanoparticles using fungi and actinomycete. Current Science 85(2): 162170

30. Gole A, Dash C, Ramakrishnan V, Sainkar SR, Mandale AB, et al. 2001. Pepsin-gold colloid conjugates: preparation, characterization, and enzymatic activity. Langmuir 17(5): 1674-1679. doi: 10.1021/ la001164w

31. Vigneshwaran N, Kathe AA, Varadarajan PV, Nachane RP, Balasubramanya RH. 2007. Silver-protein (core-shell) nanoparticle production using spent mushroom substrate. Langmuir 23(13): 71137117. doi: $10.1021 / 1 \mathrm{a} 063627 \mathrm{p}$

32. Coates J. 2000. Interpretation of infrared spectra, a practical approach. Encyclopedia of Analytical Chemistry. doi: 10.1002/9780470027318. a5606 